\title{
Medial Prefrontal Cortex Supports Recollection, But Not Familiarity, in the Rat
}

\author{
Anja Farovik, Laura M. Dupont, Miguel Arce, and Howard Eichenbaum \\ Center for Memory and Brain, Boston University, Boston, Massachusetts 02215
}

There is continuing controversy about the extent to which the rodent medial prefrontal cortical area (mPFC) is functionally homologous to the dorsolateral prefrontal cortex in humans and nonhuman primates. Previous studies have compared the effects of mPFC lesions in rats to those of dorsolateral prefrontal lesions in working memory, strategy switching, and temporal ordering. None, however, has examined the role of the rodent mPFC in recognition memory, wherein, in humans, dorsolateral prefrontal damage results in a deficit in source monitoring resulting in impaired recollection. In the present study, we examined recognition memory in rats with bilateral mPFC lesions (prelimbic/infralimbic regions; ibotenic acid) using a variant of a non-match-to-sample task with manipulations of response bias that allowed for a signal detection analysis that distinguishes recollection and familiarity contributions to recognition memory. Animals with medial prefrontal lesions had a modest overall deficit in recognition with no general change in their tendency to elicit "old" or "new" responses. Signal detection analyses indicated that rats with $\mathrm{mPFC}$ damage had a curvilinear and symmetrical receiver operating characteristic (ROC) curve, compared with a curvilinear and asymmetrical ROC curve in control subjects, indicating that mPFC damage severely reduced recollection-based performance, while sparing familiarity. The recollection failure was associated with an impaired ability to reject new items (increased false alarm rate), whereas the identification of old items (hit rate) was normal. This pattern of findings is similar to that observed in humans with dorsolateral prefrontal damage and is complementary to the selective deficit in hit rate observed after hippocampal damage.

Key words: prefrontal cortex; prelimbic; rat; recognition memory; recollection; familiarity

\section{Introduction}

One of the major outstanding questions about the evolution of the mammalian cerebral cortex is whether the functions supported by the prefrontal cortex are conserved across mammalian species. Some have argued that cytoarchitectural features of the human dorsolateral prefrontal (DLPFC) region are hardly developed in rodents, whereas others interpret connectional and neuropsychological evidence as indicating that the rat medial prefrontal cortex (mPFC) is the homolog of the primate DLPFC (Kolb, 1990; Preuss, 1995; Uylings et al., 2003; Vertes, 2006). Resolution of the controversy can be advanced by studies that extend to rodents the examination of key functions attributed to the dorsolateral prefrontal area in humans.

Numerous studies indicate that the human and nonhuman primate DLPFC supports “executive” functions including working memory (Goldman-Rakic, 1987), attentional set (Dias et al., 1996) and inhibitory control (Shimamura, 1995), selection of appropriate rules (Wise et al., 1996; Miller, 1999), temporal ordering (Milner et al., 1985; Fuster et al., 2000; Fuster, 2001), and organization and monitoring of information (Johnson et al.,

Received Aug. 3, 2008; revised 0ct. 22, 2008; accepted 0ct. 27, 2008

This work was supported by National Institute of Mental Health Project Grant MH52090. We thank Magdalena Sauvage, Norbert Fortin, and Jonathan Robitsek for helpful comments.

Correspondence should be addressed to Howard Eichenbaum, Center for Memory and Brain, Boston University, 2 Cummington Street, Boston, MA 02215. E-mail: hbe@bu.edu.

D0I:10.1523/JNEUROSCI.3662-08.2008

Copyright $\odot 2008$ Society for Neuroscience $\quad 0270-6474 / 08 / 2813428-07 \$ 15.00 / 0$
1993; Petrides, 1996). Some of these functions have been studied in rodents, and the results indicate that the rodent $\mathrm{mPFC}$ is involved in working memory (Eichenbaum et al., 1983; Granon et al., 1994), attentional control in strategy switching (Ragozzino et al., 1999; Birrell and Brown, 2000; Rich and Shapiro, 2007), and temporal organization of behavior (Kesner, 2000). One function that has not been the subject of comparative studies is the role of the rat $\mathrm{mPFC}$ in information processing that contributes to recollective memory. Yet, this particular function is a classic aspect of executive function that underlies higher-order reasoning and decision making. Recollection requires retrieval of the details and context of specific experiences, and may be dissociated from recognition based on a sense of familiarity.

Damage to the dorsolateral prefrontal area in humans results in recollection deficits (Janowsky et al., 1989; Gershberg and Shimamura, 1995; Alexander et al., 2003) but generally preserves item recognition (Swick and Knight, 1999). Furthermore, abnormally high false alarm rates (i.e., misidentification of a new item as if it was old) typically follow prefrontal lesions (Parkin et al., 1996; Schacter et al., 1996; Curran et al., 1997; Swick and Knight, 1999) and are thought to reflect misattributions of familiarity to false recollections (Kahn et al., 2004) via impaired source monitoring (Johnson, 1997).

Here, we examine the role of the rodent $\mathrm{mPFC}$ in recognition memory using a newly developed behavioral task that allows us to distinguish the contributions of recollection and familiarity to recognition performance, similar to studies that have made this 


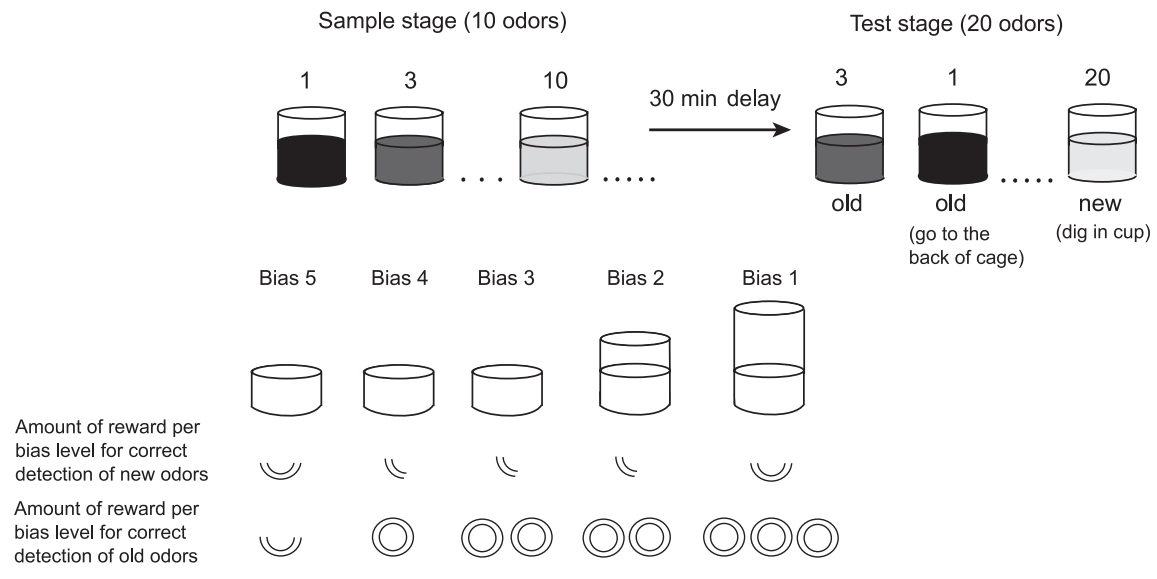

Figure 1. Recognition memory task. During the sample phase, animals were presented with 10 different odors, each baited with a Froot Loop reward. Then, after a 30 min delay, in the test phase, subjects were required to distinguish the 10 odors that were presented during the sample stage from 10 new odors that had not been presented previously on that day. If a test odor was new, the animal could dig in the cup to retrieve the reward. Conversely, if a test odor was old, the animal was required to withhold digging in the test stimulus cup and instead approach an empty cup in the back of the home cage to obtain reward. The order of old and new test odors was randomized. Response bias was manipulated by varying the height of the cup and the amount of reward (number of Froot Loops) received for responding or withholding responses to the target cup.

dissociation in humans (Yonelinas, 1994). The findings indicate that damage to the rodent $\mathrm{mPFC}$ results in a selective deficit in recollection that can be attributed to elevation of the false alarm rate, consistent with a failure of source monitoring.

\section{Materials and Methods}

Animals. Subjects were 16 male Long-Evans rats (Charles River) weighing between 225 and $250 \mathrm{~g}$ at the start of the experiment. All animals were single housed and maintained on a $12 \mathrm{~h}$ light/dark cycle (lights on 8:00 A.M. to 8:00 P.M.). Behavioral training and testing were conducted during the light phase. Animals were kept at $\sim 85 \%$ of their ad libitum feeding body weight and had ad libitum access to water in the home cage. Procedures were conducted according to the requirements set by the National Institutes of Health and Boston University Institutional Animal Care and Use Committee.

Materials and apparatus. Behavioral training and testing were performed in the home cage $(44 \times 21 \times 20 \mathrm{~cm})$. The materials consisted of transparent Nalgene cups (VWR) in three different sizes (4.0, 6.5, and 8.5 $\mathrm{cm}$ high) with an internal diameter of $\sim 6.5 \mathrm{~cm}$. Each cup was filled with playground sand and scented with one distinct odor. A selection of forty different odors was used in the study. The cups were attached to a black Plexiglas platform using Velcro (VWR) before they were lowered into the cage. Cereal Froot Loops (Kellogg's) were used as reinforcement.

Behavioral protocol. The study used a variant on the delayed nonmatching-to-sample task that characterized recognition performance over a range of response criteria (Fortin et al., 2004). The animals were required to distinguish between old and new odors using a match/nonmatch procedure in which the animal had to dig in the cup if the odor was new, and refrain from digging and go to an empty cup in the back of the cage if the odor was old. Hits were defined as correct identification of an old odor, and false alarms were defined as incorrectly responding to a new odor as if it was old. Variations in response biases by manipulations of reward payoffs and effort (Fig. 1) allowed for a dual-process signal detection analysis (Yonelinas, 1994) to distinguish recollection and familiarity contributions to recognition memory (for model equation, see supplemental material, available at www.jneurosci.org).

Animals were trained in successive stages. Initially, rats were trained to dig for reward (one Froot Loop) buried in a cup filled with unscented sand. Once the animals had learned to dig reliably to retrieve the reward, they were introduced to an odor recognition task in which each trial consisted of a sample and a test phase. In the sample phase, animals were presented with a cup filled with sand and one of the 40 odors in the front of the cage. After a 1 min delay, two cups, each with a different odor (one old and one new), were presented consecutively and in a pseudorandomized order. For new responses, one-quarter Froot Loop was buried in the cup and two whole Froot Loops (bias 2) (Fig. 1) were provided in the back of the cage for correct "old" responses. Trials that required the same response ("new" or "old") did not occur more than three times in a row during the test phase. For new trials, responses were defined as the animal moving the sand with the forepaw. Once the animals reached a criterion of $80 \%$ correct across two consecutive sessions (each session consisted of 10 trials; $5.13 \pm 1.02 \mathrm{SD}$ sessions to reach criterion), each session consisted of a series of five sample odors, and then a $1 \mathrm{~min}$ delay, and then five old and five new odors in pseudorandom order during the test phase. After the same performance criterion was reached (15.25 $\pm 5.16 \mathrm{SD}$ sessions to criterion), each session consisted of a set of 10 sample odors and 10 old and 10 new test odors, and the delay was increased to $10 \mathrm{~min}(12.13 \pm 2.89$ $\mathrm{SD}$ sessions to criterion). Odors were selected such that all 40 were used equally often, and different cups were used for odors when they appeared in both the sample and test phases. Finally, five distinct response biases were introduced by manipulating height of the cup, and therefore the effort required digging for reward, and the amount of reward obtained for correct old and new responses (Fig. 1). Animals were habituated to the different response biases, and receiver operating characteristic (ROC) analysis was performed on the 30 preoperative and 30 postoperative sessions (six sessions per bias level), using a 30 min delay period between study and test.

Surgery. Anesthesia was induced by inhalation of 5\% isoflurane (Webster Veterinary Supply) in oxygen and was maintained at $2-2.5 \%$ throughout surgery. Rats were placed in a stereotaxic frame (Kopf), and an incision was made along the midline to expose the skull. Using a $1 \mu \mathrm{l}$ Hamilton syringe, eight rats were given four injections each of $0.2 \mu \mathrm{l} /$ site of $0.06 \mathrm{~m}$ ibotenic acid (Tocris Cookson) into the prelimbic region (PL). Each injection of $0.2 \mu \mathrm{l} /$ site lasted for a duration of $3 \mathrm{~min}$ and was made using a microsyringe pump (World Precision Instruments). The needle was left in situ for 4 min after injection to allow for diffusion. Lesions were made using coordinates from the atlas of Paxinos and Watson (1998): from bregma, anteroposterior (AP), +3.5 mm; mediolateral (ML), $\pm 0.6 \mathrm{~mm}$; dorsoventral (DV), $-3.3 \mathrm{~mm}$ (from dura); and AP, $+2.7 \mathrm{~mm}$; ML, $\pm 0.6 \mathrm{~mm}$; DV , $-3.6 \mathrm{~mm}$. Diazepam ( $5 \mathrm{mg} / \mathrm{ml}$; Webster Veterinary Supply) was given intramuscularly immediately after surgery to prevent convulsions. Another eight rats (controls) underwent the same procedure as the lesion group, but saline was infused instead of ibotenic acid. After surgery, general health was monitored until they recovered and returned to testing, $\sim 1$ week after surgery.

Histology. After completion of behavioral testing, rats were overdosed with $0.8 \mathrm{ml}$ of sodium pentobarbital (Fort Dodge Animal Health). Animals were then perfused transcardially with $0.9 \%$ saline, followed by $10 \%$ formalin (VWR), and the brains were removed and placed in a $20 \%$ sucrose solution until processed. Using a cryostat (Reichert-Jung; Kramer Scientific), brains were cut into $50 \mu \mathrm{m}$ coronal sections and mounted onto presubbed glass slides, and stained with cresyl violet to determine the location and extent of the lesion.

\section{Results \\ Anatomical observations}

Figure 2 shows the area of damage at three coordinates relative to bregma [photomicrographs of PL/infralimbic region (IL) in a control and lesioned animal are provided in supplemental Fig. 1, available at www.jneurosci.org as supplemental material]. One animal was excluded from any postsurgery analysis because of major unilateral damage to the M2 and M1 cortices, leaving seven 
animals in the lesioned group and eight animals as controls. Histological quantification showed that the lesioned animals lost, on average, all of the PL/IL cortices at $3.20 \mathrm{~mm}$ anterior to bregma, whereas $81 \%$ of the total volume of the prelimbic and infralimbic cortices was damaged at 2.20 $\mathrm{mm}$ from bregma. At the most anterior coordinates ( $4.70 \mathrm{~mm}$ from bregma), the PL remained mostly intact. Five of the lesioned animals also sustained some damage to the Cg1 region and damage to the $\mathrm{Cg} 2$ area, more posteriorly. Some damage was also evident in the M2 sector, adjacent to dorsal $\mathrm{Cg} 1$, in three animals. Finally, one animal sustained minor damage to $\mathrm{MO}$, at the most anterior coordinates.

\section{Performance}

Using a least-square model, ROC curves were created from the raw scores of hits and false alarms to estimate the contribution of recollection and familiarity to recognition performance. According to the dual-process model of recognition memory, the asymmetry of the ROC in probability space, reflected in an above zero $y$-intercept, indicates the contribution of recollection. Conversely, the degree of curvilinearity (i.e., the "bowing" of the ROC function) indicates the contribution of familiarity to recognition performance (Yonelinas, 1994). In addition, to confirm the curvilinearity and symmetry of the ROC functions, linear regressions were performed on the hit and false alarm rates transformed into $z$-scores: For item recognition, a curvilinear ROC in probability space generally becomes linear in $z$-space (Yonelinas and Parks, 2007), and a slope of 1.0 in $z$-space indicates that the probability space ROCs is symmetrical, whereas a slope of $<1.0$ indicates asymmetry (Yonelinas, 1994).

Before surgery, the ROC curve was asymmetrical (slope significantly different from 1 in $z$-space, $t_{(15)}=-8.5, p<0.05$; slope average, 0.66$)$ and curvilinear $\left(F_{\text {quad } 2,2}=102.5 ; p<0.01\right)$, indicating the presence of both recollection and familiarity as contributors to recognition performance. Animals were subsequently divided into two matched groups based on overall recognition performance (Fig. $3 A$ ). The model's parameter estimates, calculated as the mean of each rat's individual $R$ and $d^{\prime}$ estimate, were not different between groups. In addition, the hit and false alarm rates were analyzed separately using repeatedmeasures ANOVA across bias levels (1-5) as within-subjects factor, and group as the single between-subjects factor (control vs $\mathrm{mPFC}$ lesion), and the results did not reveal any group differences, indicating the groups were well matched preoperatively.

Postoperatively, rats with mPFC lesions were slightly impaired on overall recognition performance compared with controls $(66 \%$ correct for controls and $60 \%$ correct for mPFC lesioned rats; $\left.t_{(13)}=2.63, p<0.05\right)$. To test whether prefrontal lesions caused a shift in the general tendency to elicit old versus new responses, we measured the overall rate of responding to the target stimulus as the combination of misses (incorrect responses to old target odors as if they were new) and correct rejections (correct responses to new target odors), both of which involve responding to the target test cup. This analysis revealed no increase in the overall tendency to respond old or new (mPFC, 36\%; controls, $41 \% ; t_{(13)}=-1.99$, NS).

In control animals, the ROC curve continued to reflect the contribution of both recollection $(R=0.38)$ and familiarity $\left(d^{\prime}=\right.$ 0.50 ) with a function that consisted of both asymmetrical (slope significantly different from 1 in $z$-space, $t_{(7)}=-5.5, p<0.05$; slope average, 0.67$)$ and curvilinear $\left(F_{\text {quad 2,2 }}=276.9 ; p<0.005\right)$ components (Fig. $3 B$ ) (for linear trends in $z$-ROC space over the range of biases tested for each group, see supplemental Fig. 2, available at www.jneurosci.org as supplemental material). In contrast, the ROC function in rats with mPFC lesions became nearly symmetrical with a slope that was not significantly different from 1 in $z$-space $\left(t_{(6)}=-2.14\right.$, NS; slope average, 0.85$)$ and a $y$-intercept value close to $0(R=0.03)$, indicating that recollection had strikingly decreased. However, the ROC function of animals with mPFC lesions preserved the curvilinear component $\left(F_{\text {quad } 2,2}=80.3 ; p<0.05\right)$, and the familiarity index $d^{\prime}$ remained significantly different from $0\left(d^{\prime}=0.49 ; t_{(6)}=3.83, p<0.05\right)$, indicating that familiarity continued to contribute to recognition 

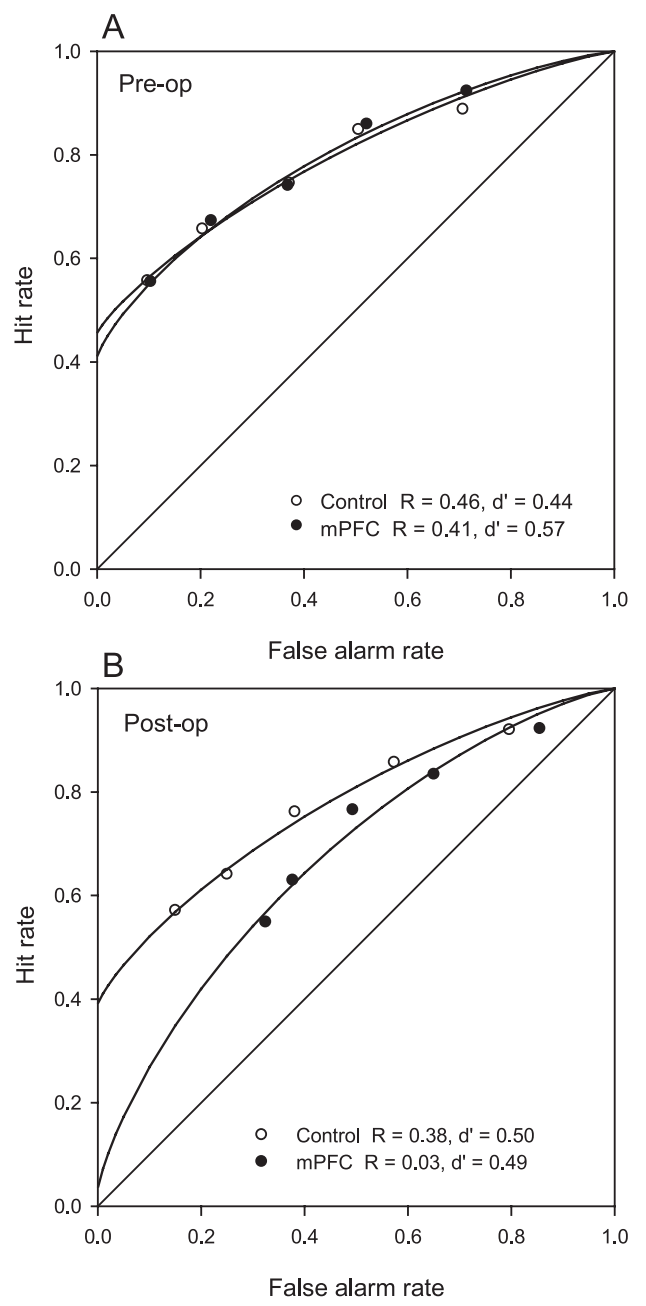

C

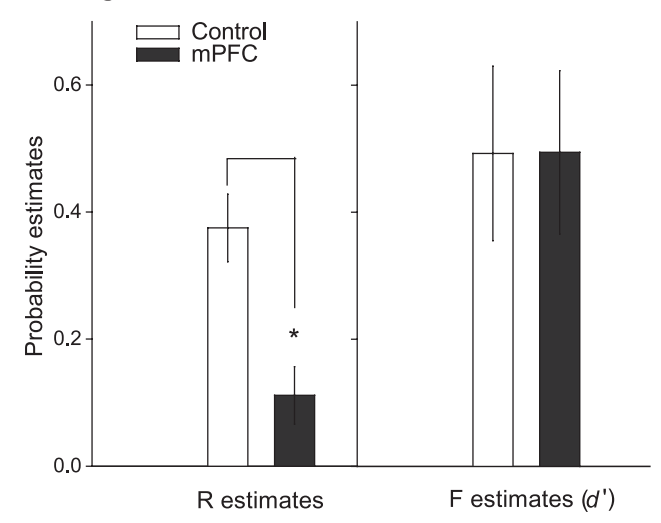

Figure 3. ROC function in recognition performance for control subjects and rats with medial prefrontal damage. $\boldsymbol{A}$, ROC for control and $\mathrm{mPFC}$ animals before surgery. $\boldsymbol{B}$, Postoperatively, control animals continued to exhibit an asymmetrical and curvilinear ROC, whereas the ROC function of rats with $\mathrm{mPFC}$ damage was curvilinear but symmetrical. Note that the rightward shifts in the scores at each bias level reflect increases in the false alarm rate at each bias. No change in the hit rate (which would be observed as the vertical displacement between corresponding scores) was evident at any bias level. C, Parameter estimates of recollection $(R)$ and familiarity $\left(d^{\prime}\right)$ for controls and lesioned animals after surgery $\left({ }^{*} p<0.05\right)$. Error bars show SEM.

performance (Fig. 3B). Comparison of the recollection and familiarity estimates in the two groups revealed no statistical difference between the two groups on familiarity $d^{\prime}$ estimates $\left(t_{(13)}\right.$ $=-0.01 ; \mathrm{NS})$, whereas estimates of recollection in animals with
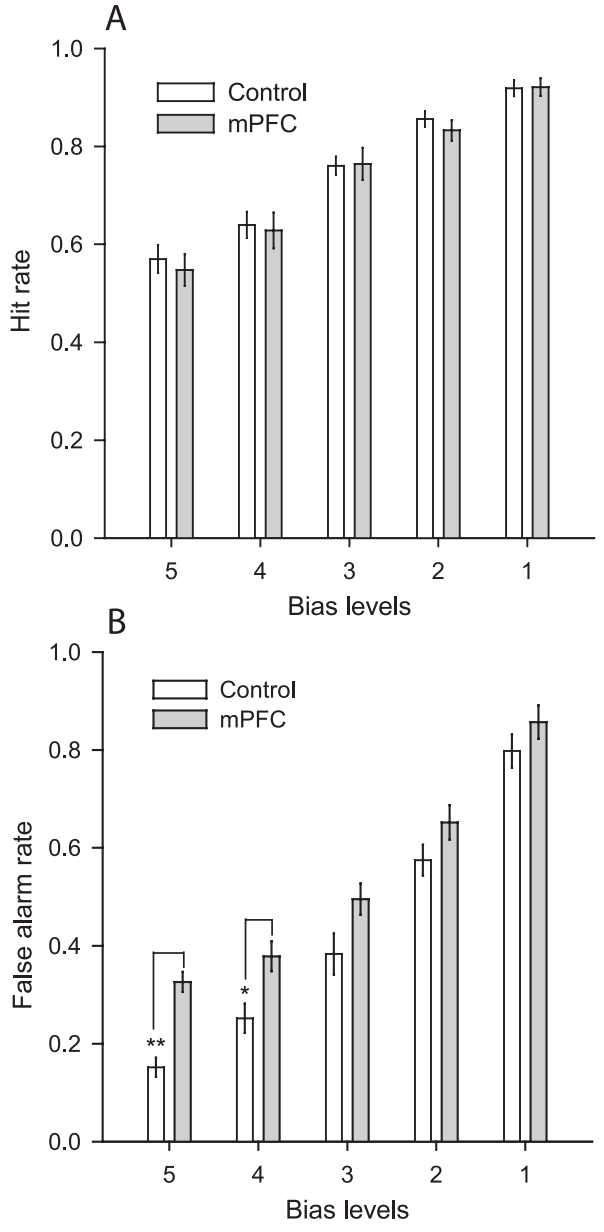

Figure 4. Hit and false alarm rates for control subjects and rats with medial prefrontal damage. The hit rates $(\boldsymbol{A})$ and false alarm rates $(\boldsymbol{B})$ are shown for each response bias level (1-5). The increase in false alarm rate was particularly large at the more conservative bias levels (bias $5,{ }^{* *} p<0.001$; bias $4,{ }^{*} p<0.05$ ). There was no effect of the lesion on the hit rate at any bias level. Error bars show SEM.

mPFC lesions were significantly lower than those for Controls $\left(t_{(13)}=3.72 ; p<0.05\right)$ (Fig. 3C).

Additional analyses on the hit and false alarm rates indicated that the recollection deficit in rats with $\mathrm{mPFC}$ lesions could be attributed to a selective increase in false recognitions of new items. Separate two-way (group by bias level) ANOVAs of hit and false alarm rates indicated that the hit rate did not significantly differ between groups $\left(F_{(1,13)}=0.12\right.$, NS; interaction group by bias level: $F_{(4,52)}=0.32$, NS) (Fig. $4 A$ ). In contrast, medial prefrontal lesions resulted in an increase in the false alarm rate compared with controls $\left(F_{(1,13)}=8.38, p<0.05\right.$; interaction of group and bias level: $F_{(4,52)}=2.78, p<0.05$ ) (Fig. $4 B$ ). Animals with $\mathrm{mPFC}$ lesions had significantly increased false alarms compared with controls at bias levels $5\left(t_{13}=-6.13 ; p<0.001\right)$ and $4\left(t_{(13)}\right.$ $=-2.96 ; p<0.05)$.

An alternative model of recognition memory, called the unequal-variance signal detection (UVSD) model, claims that recognition is based on a single process influenced by memory strength. In this model, $d^{\prime}$ reflects memory strength and the asymmetry in the ROC curve results from a larger variance in the old item distribution compared with that for new items $\left(V_{\text {old }}>\right.$ $\left.V_{\text {new }}=1\right)$. The ROC function of normal rats before surgery ( $V_{\text {old }}$ $\left.=1.53 ; d^{\prime}=1.44\right)$ and after surgery $\left(V_{\text {old }}=1.47 ; d^{\prime}=1.30\right)$ is consistent with this model. In rats with $\mathrm{mPFC}$ lesions, the vari- 
ances became equal ( $V_{\text {old }}=1.03$; not significantly different from $\left.V_{\text {new }}=1, t_{(6)}=0.38, \mathrm{NS}\right)$, and consequently only a single factor $\left(d^{\prime}=0.63\right)$ is reflected in the signal detection process. Additionally, a $\chi^{2}$ goodness-of-fit analysis showed that both models fit the data very well, with $\chi^{2}$ values considerably below the critical value, $\mathrm{df}_{(4)}=9.49$ (dual-process model: $\chi_{\text {control hits }}^{2}=0.08$, $\chi_{\text {control false alarms }}^{2}=0.06 ; \chi_{\text {mPFC hits }}^{2}=0.18, \chi_{\text {mPFC false alarms }}^{2}=$ 0.06; UVSD model: $\chi_{\text {control hits }}^{2}=0.05, \chi_{\text {control false alarms }}^{2}=0.16$; $\left.\chi_{\text {mPFC hits }}^{2}=0.11, \chi_{\text {mPFC false alarms }}^{2}=0.16\right)$, suggesting that both models can explain the obtained data. These findings also show that, even with a narrower range of false alarm scores in mPFC rats (Fig. 3B), their ROC functions are well fit by either model.

\section{Discussion}

This study examined the role of the rat $\mathrm{mPFC}$ in item recognition memory. Overall recognition performance was only modestly affected by $\mathrm{mPFC}$ damage, and the deficit was not attributable to a change in the overall tendency to elicit old or new responses. Signal detection analyses indicated that $\mathrm{mPFC}$ damage produced an almost complete loss of the contribution of recollection, whereas performance based on familiarity was unaffected. Furthermore, the recollection deficit was attributable to an increase in false recognitions of novel items with no effect on the ability to identify previously experienced items. This pattern of performance is consistent with the observation that humans with DLPFC damage have a small impairment on standard item recognition (Stuss et al., 1994) and severe deficits in recollection (Janowsky et al., 1989; Gershberg and Shimamura, 1995; Alexander et al., 2003).

Some neuropsychological studies in patients with dorsolateral prefrontal damage have also reported deficits in both recollection and familiarity (Duarte et al., 2005), as well as spared recollection and impaired familiarity (MacPherson et al., 2008). Regardless of these differences on whether DLPFC damage disproportionately impacts either recollection or familiarity, the literature is consistent in the observation that prefrontal damage results in abnormally high false alarms (Parkin et al., 1996; Schacter et al., 1996; Curran et al., 1997; Swick and Knight, 1999; MacPherson et al., 2008), similar to the current results. Interestingly, functional imaging studies have reported that different prefrontal areas are associated with recollection and familiarity (Yonelinas et al., 2005). One possible interpretation of these findings is that different parts of the prefrontal cortex are involved in distinct aspects of the recognition processing and that damage to this system can result in a shift in either recollection or familiarity judgments because of the same fundamental misattribution impairment (i.e., false recognitions).

In humans, signal detection techniques have been used in the study of recognition memory, but there is debate concerning the nature of recollection and familiarity-based recognitions. The dual-process model considers recollection as qualitatively independent from familiarity (Yonelinas, 1994), whereas the alternative view (the UVSD model) claims that familiarity and recollection differ only in memory strength, in which recollection reflects stronger memories, whereas familiarity reflects weaker memories (Wixted, 2007). In our study, the ROC functions of both the control and $\mathrm{mPFC}$ rats were well fit by both models. Nonetheless, the recollection and familiarity components of the ROC function of normal rats are doubly dissociable by variations in the memory demands and by aging (Robitsek et al., 2008; Sauvage et al., 2008), which supports a dual-process account of recognition memory in the current paradigm.

\section{False alarms and prefrontal function}

What is the mechanism by which the abnormality in false alarm rates underlies the recollection failure in rats with $\mathrm{mPFC}$ damage? One possibility is that the increase in false alarms is secondary to an inability to withhold prepotent responses to the test stimulus. An increase in anticipatory responses (i.e., impulsivity) has been shown to follow lesions of the infralimbic cortex on the fivechoice serial reaction time task (Chudasama et al., 2003; Murphy et al., 2005). A general increase in impulsive behavior would be manifested in an increased tendency to respond to the target stimulus cup, which would result in a decrease in both the hit and false alarm rates, both of which require not responding to the target stimulus in our protocol. However, the overall tendency to respond to the target stimulus was not significantly affected by $\mathrm{mPFC}$ lesions. Moreover, perseverative tendencies have also been reported after PL/IL damage (Eichenbaum et al., 1983; Passetti et al., 2002, 2003; Ragozzino et al., 2003), but a possible general change in perseveration caused by the lesion would, however, alter both hits and false alarms, and this was not found.

Another possible account of our findings is that mPFC lesions result in an inability to select the correct memory strategy. Studies indicate that rats with $\mathrm{mPFC}$ damage are unable to appropriately select between multiple alternative strategies (Birrell and Brown, 2000; Ragozzino et al., 2003; Rich and Shapiro, 2007) and competing memories (Quirk et al., 2006). Consistent with these findings, damage to the $\mathrm{MPFC}$ might result in a failure to appropriately engage hippocampal mechanisms that identify specific episodic memories of odors experienced in the current session. Alternatively, our results could be explained as a failure in source monitoring within episodic memory. In our task, odor stimuli are selected each day from a limited pool (see Materials and Methods). Therefore, each odor has on some occasion been an old item; a major challenge for the rat, then, is to distinguish the source for each odor as the current list as opposed to previous lists. A failure to assign the appropriate memory to current circumstances may underlie the high false alarm rate in rats with $\mathrm{mPFC}$ damage. Accordingly, the $\mathrm{MPFC}$ may normally contribute to recognition memory by monitoring and evaluating the source information retrieved by the hippocampus, which likely includes information from multiple experiences, and identify those odors that have the appropriate source for the current test. This possibility and that of a failure in strategy selection suggested above are not mutually exclusive, such that the prefrontal cortex may normally both engage the episodic memory system and then monitor the source of the resulting memories recovered by that system.

The role in recollection observed adds to the rat $\mathrm{mPFC}$ functions that are also commonly attributed to the DLPFC in primates. As noted by others (Brown and Bowman, 2002), the emphasis need not be placed on the relative anatomical resemblance of the prefrontal cortex across species, because the rat prefrontal region would fall short of exhibiting a corresponding biological complexity. Instead, attention should be given to the functional similarities that are becoming evident between species. There have been a growing number of behavioral studies that address the functions of the rat prefrontal cortex (for review, see Granon and Poucet, 2000), and results show remarkable functional similarities between species. The findings presented herein increase this correspondence, indicating that the rat $\mathrm{mPFC}$ and human DLPFC play comparable roles in recognition memory. 


\section{Comparison of hippocampal and prefrontal contributions to recognition memory}

Because of the range of nonmemory deficits (e.g., impulsiveness, perseveration, strategy switching) observed after prefrontal damage in humans, investigators tend to overlook the importance of considering how this structure complements and functionally interacts with the medial temporal lobe in support of memory (Buckner et al., 1999; Simons and Spiers, 2003). Studies have shown that the prefrontal cortex is involved in various aspects of memory encoding (Owen et al., 1996; Wagner et al., 1998) and retrieval (Shallice et al., 1994; Nyberg et al., 1996), suggesting that attention to the prefrontal cortex and its interactions with medial temporal lobe structures is necessary if we are to develop a complete understanding of the neural representations of recognition memory.

On an anatomical level, studies describe extensive bidirectional connections between the hippocampal region and the $\mathrm{mPFC}$ in the rat. The mPFC sends information to the medial temporal area via the midline thalamus (Vertes, 2002, 2006) and via projections to the entorhinal cortex (Deacon et al., 1983; Burwell and Amaral, 1998). Hippocampal efferents to the mPFC arise from the CA1 region and the subiculum and terminate in both infralimbic and prelimbic cortices (Jay et al., 1989; Jay and Witter, 1991). These bidirectional connections can support considerable interaction between the mPFC and hippocampus. However, the precise functional interaction between these regions in memory processes remains to be determined.

Insights about the nature of these interactions can be obtained by comparing the present results with those from previous studies in our laboratory that have examined the ROC functions of animals with hippocampal damage performing the same memory task (Fortin et al., 2004). In our previous study, damage to the hippocampus resulted in an ROC function that was curvilinear and symmetrical. In the present study, rats with $\mathrm{mPFC}$ damage exhibited the same abnormality in the ROC function, indicating that both structures play a critical and selective role in the recollection component of recognition. Additionally, in animals with hippocampal damage, the recollection deficit was accompanied by a decrease in the hit rate with a normal false alarm rate, consistent with a selective loss of memory for previously experienced stimuli. In contrast, in the present study, animals with mPFC lesions exhibited an error pattern opposite to that found in hippocampal animals. Rats with $\mathrm{mPFC}$ lesions have a normal hit rate but produce a high number of false alarms compared with controls, consistent with no deficit in retrieving memories, but an impairment in "working with memory" (Moscovitch and Winocur, 2000). Thus, the role of the rat $\mathrm{mPFC}$ and the DLPFC in humans are viewed as complementing that of the hippocampus, such that the hippocampus is considered to be essential to the retrieval of contextual and other associations of specific experiences (Brown and Aggleton, 2001; Eichenbaum et al., 2007), whereas the rat mPFC and human DLPFC are involved in monitoring the retrieved information. Together, the mPFC and hippocampus enable successful recollection of previous experiences.

\section{References}

Alexander MP, Stuss DT, Fansabedian N (2003) California verbal learning test: performance by patients with focal frontal and non-frontal lesions. Brain 126:1493-1503.

Birrell JM, Brown VJ (2000) Medial frontal cortex mediates perceptual attentional set shifting in the rat. J Neurosci 20:4320-4324.

Brown MW, Aggleton JP (2001) Recognition memory: what are the roles of the perirhinal cortex and hippocampus? Nat Rev Neurosci 2:51-61.

Brown VJ, Bowman EM (2002) Rodents models of prefrontal cortical function. Trends Neurosci 25:340-343.
Buckner RL, Kelley WM, Petersen SE (1999) Frontal cortex contributes to human memory formation. Nat Neurosci 2:311-314.

Burwell RD, Amaral DG (1998) Cortical afferents of the perirhinal, postrhinal, and entorhinal cortices of the rat. J Comp Neurol 398:179-205.

Chudasama Y, Passetti F, Rhodes SE, Lopian D, Desai A, Robbins TW (2003) Dissociable aspects of performance on the 5-choice serial reaction time task following lesions of the dorsal anterior cinculate, infralimbic and orbitofrontal cortex in the rat: differential effects on selectivity, impulsivity and compulsivity. Behav Brain Res 146:105-119.

Curran T, Schacter DL, Norman KA, Galluccio L (1997) False recognition after a right frontal lobe infarction: memory for general and specific information. Neuropsychologia 35:1035-1049.

Deacon TW, Eichenbaum H, Rosenberg P, Eckmann KW (1983) Afferent connections of the perirhinal cortex in the rat. J Comp Neurol 220:168-190.

Dias R, Robbins TW, Roberts AC (1996) Dissociation in prefrontal cortex of attentional and affective shifts. Nature 380:69-72.

Duarte A, Ranganath C, Knight RT (2005) Effects of unilateral prefrontal lesions on familiarity, recollection and source memory. J Neurosci 25:8333-8337.

Eichenbaum H, Clegg RA, Feeley A (1983) A re-examination of functional subdivisions of the rodent prefrontal cortex. Exp Neurol 79:434-451.

Eichenbaum H, Yonelinas AP, Ranganath C (2007) The medial temporal lobe and recognition memory. Annu Rev Neurosci 30:123-152.

Fortin NJ, Wright SP, Eichenbaum H (2004) Recollection-like memory retrieval in rats is dependent on the hippocampus. Nature 431:188-191.

Fuster JM (2001) The prefrontal cortex-an update: time is of the essence. Neuron 30:319-333.

Fuster JM, Bodner M, Kroger JK (2000) Cross-modal and cross-temporal association in neurons of frontal cortex. Nature 405:347-351.

Gershberg FB, Shimamura AP (1995) Impaired use of organizational strategies in free recall following frontal lobe damage. Neuropsychologia 13:1305-1333.

Goldman-Rakic PS (1987) Circuitry of primate prefrontal cortex and regulation of behavior by representational memory. In: Handbook of physiology: the nervous system (Plum F, ed), pp 373-417. Bethesda, MD: American Physiological Society.

Granon S, Poucet B (2000) Involvement of the rat prefrontal cortex in cognitive functions: a central role for the prelimbic area. Psychobiology 28:229-237.

Granon S, Vidal C, Thinus-Blanc C, Changeux JP, Poucet B (1994) Working memory, response selection, and effortful processing in rats with medial prefrontal lesions. Behav Neurosci 108:883-891.

Janowsky JS, Shimamura AP, Squire LR (1989) Source memory impairment in patients with frontal lobe lesions. Neuropsychologia 27:1043-1056.

Jay TM, Witter MP (1991) Distribution of hippocampal CA1 and subicular efferents in the prefrontal cortex of the rat studied by means of anterograde transport of Phaseolus vulgaris-leucoagglutinin. J Comp Neurol 313:574-586.

Jay TM, Glowinski J, Thierry AM (1989) Selectivity of the hippocampal projection to the prelimbic area of the prefrontal cortex in the rat. Brain Res 505:337-340.

Johnson MK (1997) Source monitoring and memory distortion. Philos Trans R Soc Lond B Biol Sci 353:1733-1745.

Johnson MK, Hashtroudi S, Lindsay DS (1993) Source monitoring. Psychol Bull 114:3-28.

Kahn I, Davachi L, Wagner AD (2004) Functional-neuroanatomic correlates of recollection: implications for models of recognition memory. J Neurosci 24:4172-4180.

Kesner RP (2000) Subregional analysis of mnemonic functions of the prefrontal cortex in the rat. Psychobiology 28:219-228.

Kolb B (1990) Prefrontal cortex. In: The cerebral cortex of the rat (Kolb B, Tees RC, eds), pp 437-458. Cambridge, MA: MIT.

MacPherson SE, Bozzali M, Cipolotti L, Dolan RJ, Rees JH, Shallice T (2008) Effect of frontal lobe lesions on the recollection and familiarity components of recognition memory. Neuropsychologia 46:3124-3132.

Miller EK (1999) The prefrontal cortex: complex neural properties for complex behavior. Neuron 22:15-17.

Milner B, Petrides M, Smith ML (1985) Frontal lobes and temporal organization of memory. Hum Neurobiol 4:137-142.

Moscovitch M, Winocur G (2000) The frontal cortex and working with 
memory. In: Principles of frontal lobe function (Stuss DT, Knight RT, eds), pp 188-209. New York: Oxford UP.

Murphy ER, Dalley JW, Robbins TW (2005) Local glutamate receptor antagonism in the rat prefrontal cortex disrupts response inhibition in a visuospatial attentional task. Psychopharmacology 179:99-107.

Nyberg L, McIntosh AR, Cabeza R, Habib R, Houle S, Tulving E (1996) General and specific brain regions involved in encoding and retrieval of events: what, where and when. Proc Natl Acad Sci U S A 93:11280-11285.

Owen AM, Milner B, Petrides M, Evans AC (1996) Memory for object features versus memory for object location: a positron-emission tomography study of encoding and retrieval processes. Proc Natl Acad Sci U S A 93:9212-9217.

Parkin AJ, Bindschaedler C, Harsent L, Metzler C (1996) Pathological false alarm rates following damage to the left frontal cortex. Brain Cogn 32:14-27.

Passetti F, Chudasama Y, Robbins TW (2002) The frontal cortex of the rat and visual attentional performance: dissociable functions of distinct medial prefrontal subregions. Cereb Cortex 12:1254-1268.

Passetti F, Levita L, Robbins TW (2003) Sulpiride alleviates the attentional impairments of rats with medial prefrontal cortex lesions. Behav Brain Res 138:59-69.

Paxinos G, Watson C (1998) The rat brain in stereotaxic coordinates, Ed 4. San Diego: Academic.

Petrides M (1996) Specialized systems for the processing of mnemonic information within the primate frontal cortex. Phil Trans R Soc Lond B 351:1455-1462.

Preuss TM (1995) Do rats have prefrontal cortex? The Rose-Woolsey-Akert program reconsidered. J Cogn Neurosci 7:1-24.

Quirk GJ, Garcia R, González-Lima F (2006) Prefrontal mechanisms in extinction of conditioned fear. Biol Psychiatry 60:337-343.

Ragozzino ME, Detrick S, Kesner RP (1999) Involvement of the prelimbicinfralimbic areas of the rodent prefrontal cortex in behavioral flexibility and response learning. J Neurosci 19:4585-4594.

Ragozzino ME, Kim J, Hassert D, Minniti N, Kiang C (2003) The contribution of the rat prelimbic-infralimbic areas to different forms of task switching. Behav Neurosci 117:1054-1065.

Rich EL, Shapiro ML (2007) Prelimbic/infralimbic inactivation impairs memory for multiple task switches, but not flexible selection of familiar tasks. J Neurosci 27:4747-4755.

Robitsek RJ, Fortin NJ, Koh MT, Gallagher M, Eichenbaum H (2008) Cognitive aging: a common decline of episodic recollection and spatial memory in rats. J Neurosci 28:8945-8954.
Sauvage MM, Fortin NJ, Owens CB, Yonelinas AP, Eichenbaum H (2008) Recognition memory: opposite effects of hippocampal damage on recollection and familiarity. Nat Neurosci 11:16-18.

Schacter DL, Curran T, Galluccio L, Milberg WP, Bates JF (1996) False recognition and the right frontal lobe: a case study. Neuropsychologia 34:793-808.

Shallice T, Fletcher P, Frith CD, Grasby P, Frackowiak RS, Dolan RJ (1994) Brain regions associated with acquisition and retrieval of verbal episodic memory. Nature 368:633-635.

Shimamura AP (1995) Memory and frontal lobe function. In: The cognitive neurosciences (Gazzaniga MS, ed), pp 803-813. Cambridge, MA: MIT.

Simons JS, Spiers HJ (2003) Prefrontal and medial temporal lobe interactions in long-term memory. Nat Rev Neurosci 4:637-648.

Stuss DT, Alexander MP, Palumbo CL, Buckle L, Sayer L, Pogue J (1994) Organizational strategies of patients with unilateral or bilateral frontal lobe injury in word list learning tasks. Neuropsychology 8:355-373.

Swick D, Knight RT (1999) Contributions of prefrontal cortex to recognition memory: electrophysiological and behavioral evidence. Neuropsychology 13:155-170.

Uylings HB, Groenewegen HJ, Kolb B (2003) Do rats have a prefrontal cortex? Behav Brain Res 146:3-17.

Vertes RP (2002) Analysis of projections from the medial prefrontal cortex to the thalamus in the rat, with emphasis on nucleus reuniens. J Comp Neurol 442:163-187.

Vertes RP (2006) Interactions among the medial prefrontal cortex, hippocampus and midline thalamus in emotional and cognitive processing in the rat. Neuroscience 142:1-20.

Wagner AD, Poldrack RA, Eldridge LL, Desmond JE, Glover GH, Gabrieli JDE (1998) Material-specific lateralization of prefrontal activation during episodic encoding and retrieval. Neuroreport 9:3711-3717.

Wise SP, Murray EA, Gerfen CR (1996) The frontal cortex-basal ganglia system in primates. Crit Rev Neurobiol 10:317-356.

Wixted JT (2007) Dual-process theory and signal-detection theory of recognition memory. Psychol Rev 114:152-176.

Yonelinas AP (1994) Receiver operating characteristics in recognition memory: evidence for a dual-process model. J Exp Psychol: Learn Mem Cogn 20:1341-1354.

Yonelinas AP, Parks CM (2007) Receiver operating characteristics (ROCs) in recognition memory: a review. Psychol Bull 133:800-832.

Yonelinas AP, Otten LJ, Shaw KN, Rugg MD (2005) Separating the brain regions involved in recollection and familiarity in recognition memory. J Neurosci 25:3002-3008. 